\section{Non-random Distribution of Bull Spermatozoa in a Drop of Sperm Suspension}

LORD ROTHSCHILD reported ${ }^{i}$ that bull spermatozoa in a liquid confined between parallel glass plates are more concentrated near to the plates, and he suggests that this might be due to a hydrodynamic interaction between the spermatozoa and the plates.

Bull spermatozoa move at about $100 \mu /$ sec and have a typical dimension of the order of $10 \mu$ so that, if the density and viscosity of the liquid are of the order of $1 \mathrm{~g} / \mathrm{ml}$. and 1 unit centipoiso respectively, Reynolds's number is of the order of $10^{-3}$. The flow may therefore be considered as creeping flow and all inertial forces may be neglected in comparison with the viscous forces.

If a body approaches a rigid plane in creeping flow the resistance to its motion towards the plane increases, because liquid has to be 'squeezed out' from between the body and the plane. The resistance to the motion away from the plane is increased by the same amount. Thus, the velocity of a spermatozoon perpendicular to the glass plates will be less when it is near to one of them than when it is in the body of the liquid. If an imaginary plano is drawn in the fluid parallel to one of the plates, the number of spermatozoa crossing in unit time from one side will be proportional to both the concentration of spermatozoa on that side and their velocity perpendicular to the plane. Under equilibrium conditions the number crossing in each direction in unit time must be the same, so that the product of concentration and speed must be a constant across the plane. An alternative way of looking at the same problem is to consider the time spent by an individual spermatozoon in different regions. This will be inversely proportional to the velocity in the region and, as this applies to each spermatazoon, the concentration in any region will be inversely proportional to the velocity across that region. Therefore it is to be expected that the concentration will be greater near to the glass plates.

The resistance to motion of a body near a rigid plane may be expressed as $F=6 \pi a V \eta \lambda$, where $\eta$ is the viscosity of the liquid, $V$ the velocity of the bo ty, and $a$ the Stokes radius, which is a length the value of which is a measure of the size of the body but the value of which depends also

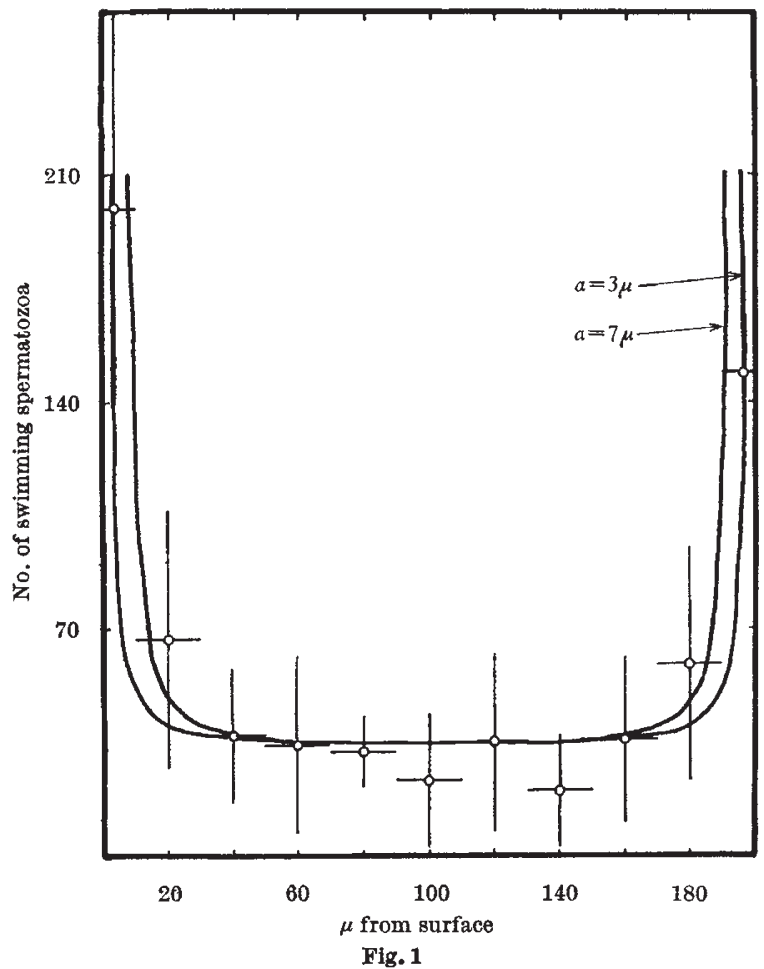

on shape. $\lambda$ is a function of the ratio of $a / b$ (where $b$ is the distance of the body from the rigid plane) which will also depend on shape. For a constant driving force, $V$ is inversely proportional to $\lambda$, so that the equilibrium concentration will be directly proportional to $\lambda$.

It may be shown that:

$$
\lambda=\left(1-\frac{9}{8} \frac{a}{b}\right)^{-1}+0\left[\left(\frac{a}{b}\right)^{2}\right] \text { (ref. 4) }
$$

regardless of the shape of the body, and that $\lambda$ becomes infinite as $1 / x$, where $x$ is the distance of the point on the body nearest to the plane from the plane. These considerations apply for a fixed orientation of the body and, in the present instance, a process of averaging over various orientations is necessary. A full calculation of $\lambda$ would be extremely difficult so that, as an approximation, it is assumed that it takes the same form for a spermatozoon as for a rigid sphere. As the function will be correct for $b=0$ and for $b$ large this approximation will probably not lead to too large an error. The function $\lambda$ is known exactly

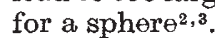

In estimating the value to be used for $a$ we may first consider the head of a spermatozoon. This approximates to an ellipsoid $1 \mu \times 5 \mu \times 10 \mu$, and a value of $3 \mu$ appears to be a reasonable value for the Stokes radius. The number of spermatozoa is taken to be $33 \lambda$ to give agreement far from the glass, and this result is plotted with the experimental results in Fig. 1. It will be seen that while the curve passes within the experimental error of all the points the agreement would have been much improved if sperma. tozoa number $=30 \lambda$ and $a=7 \mu$ had been taken. It does not seem impossible that the presence of the flagella should increase the effective radius to this amount.

While the full interaction of a spermatozoon with a rigid wall is too complicated to calculate, the proposed mechanism (which must form part of the complete interaction) could provide the hydrodynamic interaction required by Lord Rothschild.

Department of Physics,

A. D. MAUDE

University College of Wales,

$$
\text { Aberystwyth. }
$$

1 Rothschild, Lord, Nature, 198, 1221 (1963).

${ }^{2}$ Maude, A. D., Brit. J. App. Phys., 12, 293 (1961).

' Brenner, H., Chem. Eng. Sci., 16, 242 (1961).

- Maude, A. D. (to be published).

DR. MAUDE's interesting suggested interpretation of the non-random distribution of bull spermatozoa in a drop of sperm suspension may be susceptible to experimental verification. As mentioned in my original communication, other interpretations are possible. On the basis of subsequent experiments $I$ am inclined to favour the absence of elastic collisions between spermatozoa and the glass surfaces as the more probable one; but a combination of the two interpretations is not excluded. The observed distribution may have some bearing on the phenomenon of positive sperm rheotaxis.

ROTHSCHILD

Department of Zoology,

University of Cambridge.

\section{Occurrence of Ornithosis in the Wood Pigeon}

THE free-living wild birds of Britain have not been examined extensively for members of the psittacosis lymphogranuloma veneroum group. Apart from domestic pigeons living free in the cities, only herring gulls on Skomer Island have been incriminated definitely but there is also some serological evidence for infection in lesser black-backed gulls on the same Island1.

In January 1963 the opportunity arose to examine a number of adult wood pigeons (Columba palumbus) shot 